\title{
Cognitive Approach in Simulation and Control
}

\author{
Z. Avdeeva*, S. Kovriga* \\ * Institute of Control Sciences RAS, Moscow, Russia, 117997, \\ Profsoyuznaya65,e-mail:max@ipu.ru
}

\begin{abstract}
Paper presents brief review of cognitive approach in simulation and control. A class of control problems that are advisable to be solved via cognitive simulation is defined. The basic definitions are made. The method for forming strategy of ill-structured problem solution on the base of cognitive models as applies to socio-economic system is presented. Some trends of further development of cognitive approach are denoted. Copyright (C2008 IFAC.
\end{abstract}

Keywords: cognitive map, decision making, ill-structured system

\section{INTRODUCTION}

The decision theory studying control problems for systems of various nature (technical, biological, socio-economic) has become independent science branch during last decades. The decision theory actively uses methods of mathematics, psychology, informatics. The cognitive simulation is one of the new approaches of modern decision theory. This applies for studying control of ill-structured systems and situations (Narayanan and Armstrong [2005], Chaib-draa [2002], Kim [2000], Huff [1990], Kosko [1986], Sawaragi et al [1986], Heradstvein and Narvesen [1978], Axelrod [1976], Roberts [1976]) that is developed in several scientific subdivisions of Trapeznikov Institute of Control Sciences RAS (Avdeeva et al. [2007], Avdeeva [2006], Avdeeva et al. [2003], Kuznetsov et al. [2006], Kulba et al. [2004], Maximov and Kornoushenko [2001], Maximov [2001], Abramova et al. [1999]). Talking about effective control methods for complex systems, academician I.V. Prangishvili highlighted the cognitive simulation for solving of ill-structured problems that are frequently encountered in control of complex systems among practically justified scientific methods for increasing control effectiveness in organizational, socio-economic and political systems (Prangishvili [2002], Abramova et al. [1999]).

The main purpose of this paper is to form complete idea of cognitive approach actively developing now in the control science and interdisciplinary sciences (sociology, economy, etc.), further specification of basic concepts of this approach in control science, as well as defining a class of control problems that are advisable to be solved by cognitive simulation.

\section{BRIEF HISTORY OF COGNITIVE APPROACH EVOLUTION}

Origins of concept "cognitive map" lie in psychology (Tolman [1946]). Studying cognitive maps - subjective representations of spatial organization of outer world - has gained in fundamental importance in the framework of studying features of human perception of his surroundings. The cognitive map is the concept concerning cognitive processes related to gathering, representation, and processing of information on the environment during which an individual is not only a passive observer, but actively interacts with his environment. Forming cognitive maps by an individual is understood as the process consisting of series of psychological transformations. By means of these transformations, an individual gathers, stores, copies, recalls and manipulates information on relative locations and attributes of his spatial surroundings. This process is essential component of decision making for spatial behaviour. The psychological research is directed to a greater extent toward studying these very processes and their influence onto forming certain representations allowing an individual to act and make decisions in his environment.

In political science and sociology, the method of cognitive simulation was developed in 1960-1980 in papers of American researcher R. Axelrod and his colleagues from USA and Scandinavia (Heradstvein and Narvesen [1978], Axelrod [1976]).

It should be noted that many authors also use the term "cognitive mapping". In any case, research of problem situation is based on model construction on the base of a cognitive map. The differences consist only in applied modifications of cognitive maps and methods of their formal processing.

In political science and sociology, "cognitive map" is not related to spatial orientation. It is interpreted as an individual's schematic representation of world image fragment relating to concrete problem situation. In this context, cognitive map is the way for representing thought structures directed toward a concrete problem and allowing to simulate politician's mentation during deliberation of action stimulating identification of further events (Heradstvein and Narvesen [1978]).

Construction and analysis of cognitive maps allow revealing causal structure of reasoning presented in political texts and, 
on this base, making conclusion on text author's vision of political situation, defining factors taken into account by politicians while making decisions.

Axelrod developed the method of cognitive simulation relying on ideas of psycho-logic (Abelson and Rosenberg, [1958]), causal deduction (Shapiro and Bonham [1973]), as well as graph and decision theories (Axelrod [1976]). As the main problems of decision making, he distinguished the problems of situation explanation and test of hypothesis on situation arrangement, as well as prediction problems and problems of decision selection from a number of alternatives. On the base of these studies it was shown that in complex situations an individual is inclined to simplify situation representation, to not notice feedbacks, etc. As a consequence, long-term effects, interrelations between different problems, etc., are not taken into account while making decisions.

Axelrod placed the models constructed on the base of cognitive maps among normative models in that sense that they organize cognitive activity of an individual during the process of decision making. Just as any formalization, the cognitive map and methods of its analysis prescribe an individual his way of making decisions in complex situations.

The methods of cognitive simulation have found an application in collective working out and making decisions (Hart [1976], Roberts [1976]). English scientist K. Eden developed general approach to construction of cognitive maps (Eden [1988]), resting on research in the field of decision making psychology, in particular, on the theory of personal constructs by Kelly [1955]. Eden outlined importance of Kelly's statement to the effect that effectiveness of interaction in group of individuals making decisions essentially depends on each member's understanding of ways for situation interpretation by other group members.

Empirical studies of some authors (Eden [1988], Axelrod [1976], Hart [1976], Holsti [1976], Roberts [1976], Shapiro and Bonham [1973]) shown that application of cognitive simulation methods allows increasing effectiveness of decision making in problem situation. Analyzing his own and other's cognitive maps, an individual can define problem representation more exactly, find contradictions, understands other individuals. In addition, the cognitive map is the convenient tool for changing settled stereotypes contributing to generation of new viewpoints.

At the same time, Axelrod notes drawback of formal methods for construction of cognitive maps directed toward reliability and interpretability of results of problem situation analysis (Axelrod [1976]).

As a rule, application of cognitive simulation methods in sociology and political science is aimed to revealing representations of an individual making decisions in various situations, resolution of subjective conflicts due to difference between problem representations and lack of mutual understanding among interacting individuals.
The development of cognitive simulation methods is substantially conditioned by need in analysis of ill-structured systems and situations including multitude of elements with different nature, as well as relationships between elements having both quantitative and qualitative nature. The concept "ill-structured problem (situation)" was introduced by G. Simon (Simon [1973]).

The cognitive approach to analysis of ill-structured situations was proposed by Axelrod [1976] and Roberts [1976]. Axelrod to a greater extent was engaged in development of methodology, while Roberts - in development of mathematical tool. The primary precondition for rising this approach consists in boundedness of accurate model applicability to ill-structured system behaviour analysis, as well as making control decisions on resolution of problem situations.

For this approach, the subjective understanding and individual's representation of the controlled system parameters and relationships between them in form of cognitive map consists foundation for construction of illstructured model of system or situation. Subsequent choice of formal methods for processing of representations reflected in the cognitive map depends on goals of analysis, as well as characteristic features of ill-structured system or situation.

Nowadays, the cognitive approach to analysis of illstructured systems and situations is actively developed in Russia and abroad (Avdeeva et al. [2007], Kuznetsov et al. [2006], Kulba et al. [2004], Avdeeva et al. [2003], Chaibdraa [2002], Maximov [2001], Maximov and Kornoushenko [2001], Kim [2000], Huff [1990], Kosko [1986], Sawaragi et al [1986], Heradstvein and Narvesen [1978], Roberts [1976]). One of the typical trends of this development consists in looking for mechanisms joining various scientific areas of researching decision problems for control of ill-structured systems and situations.

The distinctive feature of cognitive approach consists in the following:

- Process of solving of control problems is considered as cognitive human activity including application of formal models and methods as a part or stage of solution.

- Another important stage is formalization of representation of ill-structured systems and situations, goals, interests and motivations of individuals involved into the problem solution processes.

\section{CLASS OF CONTROL PROBLEMS ADVISABLE TO BE SOLVED VIA COGNITIVE SIMULATION}

In the control science, the traditional theoretical methods focus on finding optimal solution from fixed set of alternative solutions for achieving well-defined goal.

Issues of problem identification, forming of goals and set of alternatives of their achievement often remain apart.

In real control situations, one can often face the problem consisting in not only and not so much in making the choice between alternative solutions as in analysis of situation for 
revealing real problems and causes of their appearance. Understanding of problem is indispensable precondition for finding admissible solution. At that, ill-conditioned systems are characterized by problems that hardly can be extracted from analyzed control situation. This limits applicability of traditional methods for finding optimal (or even satisfactory) solution to control problems for such systems.

One of the causes is lack of information on condition of illstructured system in conditions of weakly controlled and varying environment.

Lack of sufficient knowledge on the system as respects to which the decision is made is not a unique uncertainty conditioned by subjective causes. Uncertainty of development goals of ill-structured system and criteria for choosing control solution can also be noted. As a rule, dissatisfaction with current condition of system is realized by an individual, but his knowledge of causes and possible means of changing situation in ill-structured system are fuzzy and conflicting. Formalization of fuzzy representations is one of the main problems that have to be solved while developing models and methods of making decisions in ill-structured situations.

It is also important to take into account that the subject of control very often has to make decisions in permanently varying conditions and limited endurance.

Another difficulty consists in that the subject of control has to manipulate qualitative information in form of hypotheses (assumptions), intuitive concepts, and semantic images. Numerous studies of decision making processes confirm that the subject of control is unusual for thinking and making decisions only in quantitative terms. He thinks mainly qualitatively and sees the solution finding process as, first of all, searching of solution idea, where qualitative estimations play auxiliary role. Therefore knowledge structures in thought of the subject appear to be the most important elements of situation that cannot be reduced because of model of decision making.

The characteristic feature of ill-structured system analysis consists in that the process of preparation and making decisions on control of ill-structured system is, as a rule, a group activity. Each participant of this process represents problem situation based on "his own" inherent representations and knowledge of situation (images, models of world). An image of world includes a set of convictions, perceptual features, cost and practical rules of an individual that guide his activity and influence the process of problem situation resolution.

Thus, decision preparation and making in control problems for ill-structured systems should be considered as complex intellectual process of problem resolution that cannot be reduced to solely rational choice.

Supporting this process requires new approaches to development of formal models and methods for both problem solution and forming goals of ill-structured system development, especially at early stages of preparation of control decisions. Papers by Larichev and Moshkovich
[1997] denote that the first stage with application of methods for making decisions - "preliminary analysis of problem and its structuring" - is the most complex and hardly formalized. At this stage, "experienced advisers and analysts" are engaged in work, and arsenal of applied methods, as a rule, includes heuristic expert methods (brainstorm, interviewing, etc.).

The cognitive approach to simulation and control of illstructured systems is aimed at development of formal models and methods supporting intellectual process of problem solution due to taking into account human factor and knowledge accumulated and developed in cognitive science.

\section{BASIC CONCEPTS AND MODELS IN MODERN COGNITIVE SIMULATION}

The key concepts that have become prevalent and are widely used within the framework of cognitive approach and its various branches are rarely defined in publications. At that, ambiguity of comprehension occurs quite often right up to contradictions due to different understanding both in different branches, and, in some cases, inside of one branch. We try to define more accurately several basic concepts of cognitive approach to solving problems of analysis and control of illstructured systems and situations.

This section also briefly considers the main types of mathematical models used in modern cognitive approach to solving problems of analysis and control of ill-structured systems and situations.

The cognitive simulation in problems of analysis and control of ill-structured systems is the research of operation and development of ill-structured systems and situations via construction of ill-structured system (or situation) model on the base of cognitive map.

Within the framework of forming of cognitive approach in the control science, the term "cognitive map" is understood in a wide sense, i.e. it is not connected to representations within a context of spatial orientation, as is customary in psychologic studies (see Section 2).

The cognitive map (Kuznetsov et al. [2006], Maximov and Kornoushenko [2001], Maximov [2001]) reflects subjective representations (individual or collective) of considered problem or situation related to operation and development of ill-structured system. The basic factors and causal relations between them are the main elements of the cognitive map (Kuznetsov et al. [2006], Maximov and Kornoushenko [2001], Maximov [2001]).

It is important to note that a model includes the cognitive map as obligatory element along with other possible parameters. In many papers developing the cognitive approach to control "cognitive map" is often identified with cognitive model. However, one should remember that in psychologic studies the concept "cognitive model" is connected to the mental model of individual generated under the influence of individual's cognitive abilities.

Substantially, the basic factors are the factors that 
(1) define and limit observed events and processes in illstructured system and its environment and

(2) are interpreted by subject of control as essential key parameters, features of these events and processes.

In process of the cognitive approach formation, the formal representation of a cognitive map in a form of a signed graph was accepted. The signed graph is the directed graph with nodes associated with factors and ribs associated with signs ("+" or "-"). Lately, the cognitive map is more often represented in form of weighted graph with nodes associated with factors and ribs associated to weight in various scales. Therefore, it can be accepted that the cognitive map in form of signed or weighted graph over the set of factors is formally common for all studies within the framework of cognitive approach.

As it is noted by Kuznetsov et al. [2006], various interpretations of nodes, ribs and weights on ribs, as well as various functions defining influence of ties onto factors result in different modifications of cognitive maps and tools for their analysis. At that, interpretations may differ both substantially and mathematically. Owing to multitude of cognitive map modifications, one can distinguish different types of models based on these maps.

By type of used relationships, five types of cognitive maps are distinguished (Huff [1990]):

(1) Maps that assess attention, association and importance of concepts: With these maps, the map maker searches for frequent use of related concepts as indicators of the strategic emphasis of a particular decision maker or organization, for example, and look for the association of these concepts with others to infer mental connection between important strategic themes. He also might make judgments about the complexity of these relationships or differences in the use of concepts;

(2) Maps that show dimension of categories and cognitive taxonomies: The map maker investigates here more complex relationships among concepts. He might dichotomize concepts and construct hierarchical relationships among broad concepts and more specific subcategories. Maps of this type have been used to define the competitive environment, and to explore the range and nature of choices perceived by decisions makers in a given setting;

(3) Maps that show influence, causality and system dynamics (causal maps): These maps allow the map maker to focus on action, as for example, how the respondent explains the current situation in terms of previous events, and what changes $\mathrm{He}$ expects in the future. This kind of cognitive map is currently, has been, and is still, the most popular mapping method;

(4) Maps that show the structure of argument and conclusion: This type of map attempts to show the logic behind conclusions and decisions to act. The map maker includes here causal beliefs, but looks more broadly at the text, as a whole, to show the cumulative impact of varied evidence and the links between longer chains of reasoning;
(5) Maps that specify frames and perceptual codes: This approach suggests that cognition is highly conditioned by previous experience, and that experience is stored in memory as a set of structured expectations.

Applied practice shows that the maps of third type are expedient for studying of ill-structured systems. Choice of method for structuring of ill-structured systems and situations in a form of set of factors and causal relations is conditioned by that the events and processes of operation and development of ill-structured systems include various events and trends defined by many factors, at that each of them in turn has an influence on some number of other factors. The networks of causal relations between them are formed (Kuznetsov et al. [2006], Heis [1980], Dorner [1997]). Book of well-known German psychologist Dorner [1997] devoted to studying thought of control subject and analysis of causes of mistakes while resolving problem situations in operation and development of complex systems indicates that a momentary situation with its features is only actual state of system and its variables. One should not only understand events that happen, but also foresee events that will happen or may happen in the future, as well as suppose a way for situation changing depend on concrete interference. It requires structured knowledge, i.e. the knowledge of relationships and mutual influences of system variables. Dorner notes that in ideal case this knowledge is represented in the form of "mathematical functions", but in case when the latter cannot be constructed one can apply diagrams of causal relations allowing to reconstruct various assumptions (hypotheses) in mind of the subject of control, at that not in the form of "causal chains", but in the form of "causal networks".

The problems of system (situation) control can be solved on the base of the cognitive map. If we establish goal and input factors that can be affected, the circle of tasks that can be solved includes goal reachability estimation, development of scenario and control strategies, and search of control decisions.

According to Kuznetsov et al. [2006], the situation analysis problems on the base of cognitive maps can be divided into two types - static and dynamic. Static analysis or influence analysis is analysis of considered situation via studying structure of mutual influence in a cognitive map. The influence analysis chooses factors with the strongest influence onto goal factors that are the factors with values to be changed. Dynamic analysis lies in the base of generating possible scenarios of situation development with time. Thus, abilities of solution of analysis and control problems are defined by type of used models, static or dynamic.

As a rule, two types of mathematical tools are used for carrying out these kinds of analysis - linear dynamic systems and fuzzy mathematics.

Nowadays, research abroad is mainly devoted to development of models on the base of fuzzy mathematics and static models using different mathematical tools (Chaib-draa [2002], Kim [2000], Kosko [1986], Sawaragi et al [1986], Heradstvein and Narvesen [1978]). 
In Russia, along with development of methods for static analysis of cognitive maps, special efforts are directed to research of ill-structured systems and situations using linear dynamic models that are presented in this paper by works of Russian scientists Avdeeva et al. [2007], Avdeeva [2006], Kulba et al. [2004], Avdeeva et al. [2003], Maximov and Kornoushenko [2001], Maximov [2001]).

In linear dynamic model based on cognitive map, factor is formally defined as variable taking values from some numerical scale.

Change of factor value with time is given by (Kulba et al. [2004], Maximov [2001], Maximov and Kornoushenko [2001])

$$
x_{i}(t+1)=x_{i}(t)+\sum_{j \in I_{i}} a_{i j}\left(x_{j}(t)-x_{j}(t-1)\right), \quad i=1, \ldots, N
$$

where $x_{i}(t+1)$ and $x_{i}(t)$ are the values of $i$-th factor at instants $t+1$ and $t$, respectively, $x_{j}(t)-x_{j}(t-1)=\Delta x_{j}(t)$ is the increment of factor $x_{j}$ at instant $t$ characterizing the rate of change (trend) of $x_{j}, a_{i j}$ is the weight of the factor $x_{j}$ influence onto factor $x_{i}, I_{i}$ is the set consisting of numbers of factors directly effecting the factor $x_{i}$.

Book of Kulba et al. [2004] offeres an approach to stability analysis of ill-structured systems based on the method of signed and functional graphs and impulse processes over them. System stability and searching for control strategy on the base of structure modification for stabilizing simulated processes becomes the central issue of research. Within the framework of developed approach, the resonance condition occurring as a result of interaction between cycles is studied. The problem of approximation of an arbitrary directed graph by the directed graph with special structure - rose. The methodology for deriving scenarios of ill-structured system that allows carrying out research of its behaviour for various control actions is developed (Kulba et al. [2004]). Analysis of process dynamics in ill-structured systems is based on assumption of possible model structure modification. A model structure modification, for example, establishing or removing relationships between factors, is connected with considering constraints taking into account the ability of subject interpretation of obtained solutions.

Experience of applying models constructed on the basis of cognitive maps for solving ill-structured problems has shown that in some cases the structure modification is hardly interpreted in terms of object domain. On the other hand, papers of Avdeeva et al. [2007], Avdeeva [2006], Avdeeva et al. [2003], Maximov [2001], Maximov and Kornoushenko [2001] are to the greater extent aimed at analysis of stable process in the model, because stable processes allow correct subject interpretation of studied events (Maximov [2001], Maximov and Kornoushenko [2001]). Initial rates of factor change (initial trends) characterizing prehistory of change of considered situation are assigned as input parameters. The values of factors are not properly fixed, that is the dynamics of situation changes is simulated on the base of factor increments. This research group focuses special attention to the following:
(1) Searching and development of structuring methods aimed at construction of cognitive maps.

(2) Increasing technological effectiveness of scientific and instrumental support for solving practical control problems.

This research group has developed the following methods and approaches (Avdeeva et al. [2007], Avdeeva [2006], Avdeeva et al. [2003], Maximov and Kornoushenko [2001]):

- Method of structure and goal analysis of ill-structured system development.

- Approach to studying conflict situations generated by contradictions in interests of subjects influencing development of considered system.

- Approach and methods for solving ill-structured problems and deriving scenarios of ill-structured system development.

At that, the problem is defined as discrepancy between current condition of ill-structured system and its dynamic and desired condition that is given by the subject of control. Complex application of the mentioned methods allows carrying out static and dynamic analysis while studying illstructured systems.

\section{METHOD OF DERIVING OF STRATEGY FOR PROBLEMS SOLVING}

The socio-economic systems constitute a typical class of illstructured systems that are expedient for application of cognitive simulation for development problem solving.

In general, the control of socio-economic system can be represented as construction of strategy for the system development, defining the main goals and general directions for their reaching, and its implementation.

Revealing the system development problems influencing negatively achievement of strategic control goals is one of the key stages of construction of strategy for socio-economic system control.

This section presents the general scheme of method for forming solution strategy for ill-structured problems on the base of linear dynamic models on the base of cognitive map in regard to socio-economic systems (Fig.1).

The control problem consists in transfer of socio-economic system into one of the states corresponding to goal image. At that, the proposed approach allows determining the system state in both values of model factors and rates of factor changes.

In the variant of approach described in this paper the state of socio-economic system is defined by rates of change of model factors. Correspondingly, the dynamics of change in simulated situation is analyzed on the basis of factor trends. 


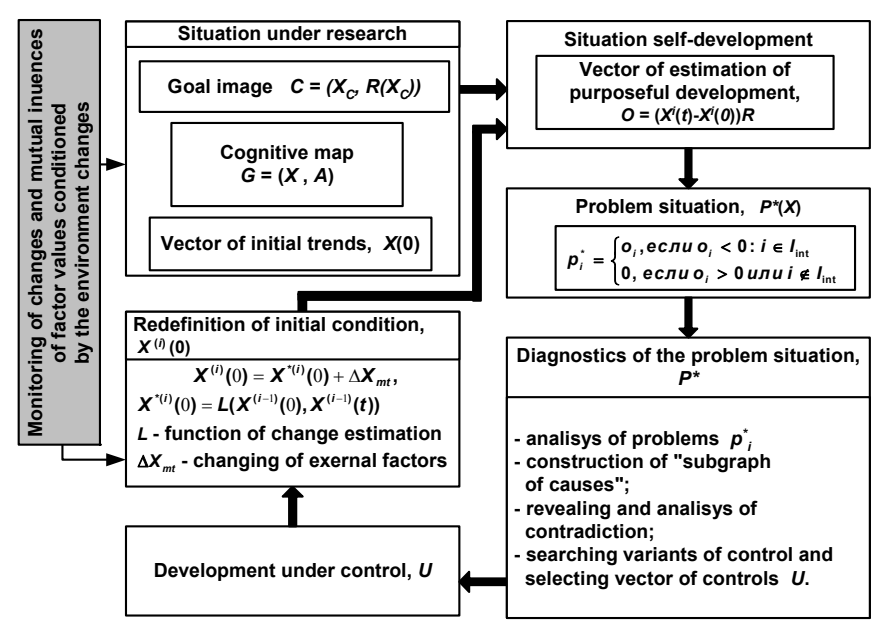

Fig. 1. General scheme of method for forming strategy for illstructured problems solution

The goal image of socio-economic system defines desirable directions of changing of the system state from the position of control subject. Formally, it is represented as

$$
C=\left(X^{C}, R\left(X^{C}\right)\right)
$$

where $X^{C}$ is the subset of goal factors, $X^{C} \subseteq X$ ( $X$ is the set of model factors), $R\left(X^{C}\right)$ is the vector of estimations of factor dynamics defining desirable directions for changing of goal factors:

$$
R\left(x_{i}^{c}\right)=\left\{\begin{array}{l}
+1, \text { if acceleration of } x_{i}^{c} \text { changing rates } \\
\quad \text { is desirable, } \\
-1, \text { otherwise. }
\end{array}\right.
$$

The strategy for socio-economic system development problems solution consists of $w$ strategic steps that define the sequence of system state changing

$$
S^{0} \rightarrow S^{1} \rightarrow \mathrm{S}^{2} \ldots \rightarrow \mathrm{S}^{m} \rightarrow S^{C},
$$

where $S^{0}$ is the initial state, $S^{C}$ is the goal state (corresponding to goal image (2)), $S^{i} \rightarrow S^{i+1}$ is the strategic step, at which the problem is revealed, and, on the base of its analysis, from the set of model factors $X$, the subset of local goals (goal factors) and the subset of controls (control factors) are selected. At that, changing of control factors results in desirable changing of goal factors.

Each strategic step $S^{i} \rightarrow S^{i+1}$ includes the following:

- Revealing the problem on the base of self-development simulation for initial state at $i$-th step (Maximov [2001], Maximov and Kornoushenko [2001]). As a result, the problem becomes defined more precisely in form of the subset $P^{i}$ of factors, which changes do not correspond to the goal image.

- Diagnostics of the problem via construction of "subgraph of causes" and structure and goal analysis (Avdeeva [2006], Avdeeva et al. [2003], Maximov and Kornoushenko [2001]) with the purpose of extracting the subset of local non-contradictory factors $Y^{i}$ from $P^{i}$ and searching variants of control (subsets of control factors) $U_{j}^{i}$ facilitating change of $Y^{i}$ in desired direction.
Non-contradiction of the goal factors means that the desired change of any goal factor from $Y^{i}$ does not result in undesirable change of other factors form $Y^{i}$.

- Simulation of the controlled system development consisting the base for forming various scenarios of the controlled development for resolving the problem applying obtained variants of control $U_{j}^{i}$ and carrying out the comparative appraisal of scenarios with the purpose of selecting control being optimal for this strategic step (Avdeeva et al. [2007], Avdeeva [2006], Avdeeva et al. [2003], Maximov [2001]).

- Redefinition of initial condition for the next strategic step via transformation $L\left(S^{i}\right)$ of the resulting state factor values $S^{i}$ at current step (Avdeeva [2006]) taking into account results of monitoring (control) of changes and mutual influences of factor values conditioned by the environment changes (Avdeeva et al. [2007], Avdeeva [2006], Avdeeva et al. [2003]).

At the next step, the cycle is repeated since new initial conditions can result in new problems preventing the desired development of socio-economic system.

The strategy construction process is finished with reaching satisfactory result that consists in reaching nearest approach to the desired system state corresponding to the goal image in conditions of given constraints (invariability of model structure, limitations on control resources, etc.) (Avdeeva [2006]). This means that, starting from some strategic step, estimation of purposeful development of the system given as functional of goal achievement degree does not change.

\section{CONCLUSION. DIRECTIONS OF FURTHER RESEARCH}

Experience of applying of various models and methods on the basis of cognitive approach (in Russia and abroad), as well as increasing interest of practical specialists to developments in this directions show expediency of development of this approach in control. At that, we should note some unsolved (or partially solved) problems. Let us outline some directions of research planned by the authors within the framework of development of cognitive approach.

Development of theoretical principles, methods, and technologies for constructing models on the base of cognitive approach while studying ill-structured systems and situations. This direction supposes forming the main principles and system of criteria (Abramova [2006], Abramova and Kovriga [2006], Abramova and Kovriga [2004]) directed toward the following:

- Increasing co-ordination and mutual understanding between participants of the process of resolving complex problem situation.

- Increasing formalization authenticity for initial knowledge (representation) of the problem situation.

The general conceptual scheme for control of model construction process for ill-structured system (situation) is developed. The criteria of appropriate transition from initial representation of ill-structured system (situation) in form of 
the cognitive map to one or another mathematical model defining further formal processing of initial representations are formed. Applicability of formal model is estimated reasoning from features and specificity of considered system (situation).

The approach to formalization of initial representations of illstructured problem in form of collective cognitive map with the purpose of generalization and agreement of different representations between problem bearers, which are competent in various object areas of knowledge. Solution of this problem rests upon the developed methods of conceptual structuring (Avdeeva [2006], Avdeeva et al. [2003]), as well as criteria and particular technologies of forming and agreement of collective concepts (Abramova et al. [1999]).

The authors also plan to carry out the cycle of works on integration of cognitive approach and methods of active system theory, since both scientific directions attach great importance to studying complex systems including active subjects that essentially influence the system control effectiveness as one of the main elements.

Development of instrumental tools for support of intellectual activity of subject in control of development of ill-structured systems and situations. By now, the software analytical system implementing the functions of model construction on the base of cognitive maps, scenario simulation and comparative estimation of scenarios has been developed (Avdeeva et al. [2007], Avdeeva [2006], Avdeeva et al. [2003]). The modular architecture of the developed system allows to build it up with other tools for solving various control problems, as well as interaction with modern information and analytical systems (for example, systems of gathering and analysis of information, ERP-systems).

Further development of this software system is aimed at the development of interface providing soft intellectual control of purposeful process of generation of formalized knowledge of subject (individual and collective).

\section{REFERENCES}

R.P. Abelson, and M.J. Rosenberg. Symbolic psycho-logic: A model of attitudinal cognition. Behavioral Science, 3:113,1958

N.A. Abramova. A subject of intellectual activity under cognitive control of ambient intelligence. Proc. of the 9th IFAC Symposium on Automated Systems Based on Human Skills and Knowledge, 2006.

N.A. Abramova, S.V. Kovriga, and other. Searching approach to problem solution. SYNTEG, Moscow. 1999.

N.A. Abramova, and S.V. Kovriga. On some aspects by forming and agreement of concepts and their influence onto quality of intellectual activity. Proc. of the 2nd International Conference on Cognitive Sciences. SaintPetersburg, 2006.

N.A. Abramova, and S.V. Kovriga. On risks related to error of experts and analysts. J. Control Sciences. 6:60-67, Moscow, 2006.
Z.K. Avdeeva. Methods for forming solution strategy for illstructured problems using cognitive models. Preprint. Voronezh, VGTU, 2006.

Z. Avdeeva, S. Kovriga, and other. Goal setting and structure and goal analysis of complex systems and situations. Proc. of the 8th IFAC Symposium on Automated Systems Based on Human Skill and Knowledge. Göteborg, Sweden, 2003.

Z. Avdeeva, S. Kovriga, and other. Cognitive approach to problem solving of social and economic object development. Proc. 4th International Conference on Informatics in Control, Automation and Robotics. Angers, France, 2007.

R. Axelrod. The cognitive mapping approach to decision making. In R. Axelrod, editor, Structure of Decision. The Cognitive Maps of Political Elites. Princeton University Press, Princeton, 1976.

B. Chaib-draa, Causal maps: theory, implementation, and practical applications in multiagent environments. IEEE Trans. on Knowledge and Data Engineering. 14(6):10-27, 2002.

D. Dorner. The Logic of Failure: recognizing and avoiding error in complex situations, Perseus, Massachusetts, 1997.

C. Eden. Cognitive mapping Eur. J. of Operational Res. 36(1):1-13, 1988.

J. Hart. Comparative Cognition: Politics of International Control of the Oceans. In R. Axelrod, editor, Structure of Decision. The Cognitive Maps of Political Elites. Princeton University Press, Princeton, 1976.

D. Heradstvein, and O. Narvesen. Psychological constraints on decision-making. A discussion of cognitive approaches: operational code and cognitive map. Cooperation and Conflict. 13(2):77 - 92, 1978.

O. Holsti. Foreign policy formation viewed cognitively. In R. Axelrod, editor, Structure of Decision. The Cognitive Maps of Political Elites. Princeton University Press, Princeton, 1976.

A. Huff. Mapping strategic thought. In A. Huff, editor, Mapping strategic thought. Wiley, Chichester, 1990.

D. Heis. Causal analysis in statistical research. Finansy and Statistica, Moscow, 1980.

G.A. Kelly. The psychology of personal constructs. Vol.1: A theory of personality. Norton, New York, 1955.

D. Kim. A simulation method of cognitive maps. Proc. of 1 st International Conference on Systems Thinking in Management. Deakins University, Australia, 2000.

B. Kosko. Fuzzy cognitive maps. Int. J. of Man-Machine Studies. 1:65-75, 1986.

V.V. Kulba, D.A. Kononov, and other. Methods for forming development scenario for socio-economic systems. SYNTEG, Moscow, 2004.

O.P. Kuznetsov, A.A. Kilinich, and A.V. Markovskii. Influence analysis in control of ill-structured situations based on cognitive maps. In N. Abramova, D. Novikov, and K. Hinsberg, editors, Human factor in control sciences. URSS, Moscow, 2006.

O. Larichev, and H. Moshkovich. Verbal decision analysis for unstructured problems. Kluwer Academic Publishers, Boston, 1997.

Z.Q. Liu, and Zhang J.Y. Interrogating the structure of fuzzy cognitive maps. Soft Computing, 7:148-153, 2003. 
V. Maximov, and E. Kornoushenko. Analytical basics of construction the graph and computer models for complicated situations. Proc. of the 10th IFAC Symposium on Information Control Problems in Manufacturing, 2001.

V. Maximov. Cognitive analysis and situation modelling. In: Proc. of the 8th IFAC Conference on "Social Stability: The Challenge of Technology Development”, 2001.

V.K. Narayanan, and D.J. Armstrong. Causal mapping for research in information. Technology, Idea Group Publishing, USA, 2005.

I.V. Prangishvili. Universal systems laws of functioning of natural and social systems and problems of control. In Theoretical and methodological problems of systems approach. Nauka, Moscow, 2002

F. Roberts. Discrete mathematical models with applications to social, biological and environmental problems. Prentice Hall, New Jersey, 1976.

T. Sawaragi, S. Iwai, and other. An integration of qualitative causal knowledge for user-oriented decision support. Control Theory and Advanced Technology. 2:451-482, 1986.

M.J. Shapiro, and G.M. Bonham. Cognitive processes and foreign policy decision-making. International Studies Quarterly. 17:147-174, 1973.

H. Simon. The Structure of Ill-structured Problems. Artificial Intelligence. 4:181-202, 1973.

E.C. Tolman. Cognitive maps in rats and men. Psychological Review. 55:189-208, 1946. 